\title{
Light-induced breathing in photochromic yttrium oxyhydrides
}

\author{
Elbruz Murat Baba $\odot, 1,4,{ }^{*}$ Jose Montero $\odot, 2,{ }^{*}, \dagger$ Evgenii Strugovshchikov, ${ }^{3}$ Esra Özkan Zayim, ${ }^{4,5}$ and Smagul Karazhanov ${ }^{1}$ \\ ${ }^{1}$ Department for Solar Energy, Institute for Energy Technology, NO-2027 Kjeller, Norway \\ ${ }^{2}$ Department of Engineering Sciences, The Ångström Laboratory, Uppsala University, Uppsala SE-75121, Sweden \\ ${ }^{3}$ Institute of Physics, University of Tartu, Tartu 50411, Estonia \\ ${ }^{4}$ Nano Science \& Nano Engineering Department, Istanbul Technical University, Istanbul 34469, Turkey \\ ${ }^{5}$ Department of Physics, Faculty of Science and Letters, Istanbul Technical University, Istanbul 34469, Turkey
}

(Received 29 April 2019; revised manuscript received 13 December 2019; accepted 3 January 2020; published 14 February 2020)

\begin{abstract}
When exposed to air, metallic yttrium dihydride $\mathrm{YH}_{2}$ films turn into insulating and transparent yttrium oxyhydride (YHO). The incorporation of oxygen causes the lattice expansion of $\mathrm{YH}_{2}$ and the emergence of photochromic properties, i.e., YHO darkens reversibly when illuminated with light of adequate energy and intensity. However, the adequate bleaching of the photodarkened samples once the illumination has stopped is much faster in air than in inert atmosphere. According to this experimental evidence, the photochromic mechanism has to be related to an oxygen diffusion and exchange process. Since this process is accompanied by a lattice expansion/contraction, it can be said that YHO "breathes" when subjected to illumination/darkness cycling. Another interesting side effect of the breathing is the unexpected enhancement of the hydrophobicity of the YHO samples under illumination. A theoretical model able to explain the breathing in YHO is presented, together with the discussion of other alternative explanations.
\end{abstract}

DOI: 10.1103/PhysRevMaterials.4.025201

\section{INTRODUCTION}

Yttrium hydride and other rare-earth hydrides are extremely strong reducing agents, a feature that considerably complicates their study. For their adequate handling in air, rare-earth hydride thin films are usually protected against oxidation by, for example, Pd capping layers [1]. However, the incorporation of oxygen in rare-earth hydrides after intentional exposure to air [2-4], or even through accidental contamination [5], leads to the formation of oxyhydrides, materials that contain oxide and negatively charged hydride [6-9], which exhibit very interesting properties. One of the pioneering works on this family of materials was carried out by Miniotas et al. [5], who reported gigantic electrical resistivity in oxygen-containing gadolinium hydride. Later, Mongstad et al. [10] reported photochromic properties in oxygen-containing yttrium hydride, a feature observed very recently by Nafezarefi et al. [2] in other-rare earth oxyhydrides such as dysprosium, gadolinium, or erbium oxyhydrides.

The photochromism in Y-related compounds can be traced back to Ohmura et al. [11], who observed light-induced reversible darkening in yttrium hydride thin films subjected to high pressures $(\sim \mathrm{GPa})$. Despite the importance of the discovery, the emergence of this new inorganic photochromic

\footnotetext{
*These authors contributed equally to this work.

†jose.montero-amenedo@angstrom.uu.se
}

Published by the American Physical Society under the terms of the Creative Commons Attribution 4.0 International license. Further distribution of this work must maintain attribution to the author(s) and the published article's title, journal citation, and DOI. Funded by Bibsam. material went unnoticed at that time, presumably because the pressure range required is not suitable for practical applications. Today, however, it is known that yttrium oxyhydride, as well as other rare-earth oxyhydrides, are photochromic at room temperature and at ambient pressure; hence, yttrium oxyhydride (YHO), as an inorganic photochromic material, has a multitude of potential applications [12]. Note that in the text we refer to yttrium oxyhydride simply as YHO, a notation that, in principle, is not related to the stoichiometry of the compound, which will be discussed later.

The origin of the photochromic mechanism in YHO is still open to debate and has been attributed to different causes [13-15]. In the present paper, the study of the wettability of the YHO surface under illumination and darkness conditions, as well as the photochromic darkening/bleaching dynamics in air and in inert atmosphere, unraveled the cause that underpins the photochromism in YHO.

According to our observations, oxygen diffusion takes place during illumination (consequently, the YHO lattice contracts). The displaced oxygen atoms leave behind an oxygendeficient structure responsible for the optical darkening, which is in agreement with our previous observations [13]. In darkness, the YHO lattice expands back as a consequence of the filling of the oxygen vacancies by oxygen atoms, allowing the film to bleach back to its original state. Since YHO expands/contracts reversibly under dark/illumination cycling produced by the displacement inwards/outwards of oxygen atoms, we refer to this process as breathing.

Due to this breathing, the correct bleaching of the photodarkened YHO coatings depends on the availability of an oxygen source. Thanks to the light-induced oxygen diffusion, YHO could be used for other purposes such as sensing and optical memories, broadening the traditional fields of application of photochromic materials. 
The wettability of YHO under illumination is unusual. All oxides and nitrides of low-electronegativity metals can exhibit hydrophobicity $[16,17]$. Therefore it can be expected that YHO exhibits hydrophobic properties as well. However, while the surface of yttrium oxyhydride increases its hydrophobicity when illuminated, other metal oxides become hydrophilic under UV illumination. In the latter case, the formation of electron-hole pairs under illumination leads to the creation of defect sites where hydroxyl groups can be adsorbed, leading to hydrophilic properties [18]. Generally, when metal oxides are stored in darkness during periods of time ranging from 7 to 50 days $[19,20]$, oxygen replaces back the adsorbed hydroxyl groups, giving raise to hydrophobicity. In the present work, the unexpected behavior observed in YHO, i.e., the enhancement of the hydrophobic properties under illumination, has been found to be caused by the same reason, that is, the oxygen enrichment of the surface under illumination.

\section{METHODS}

YHO thin films were prepared onto glass substrates following a two-step deposition process. First $\mathrm{YH}_{2}$ metallic films were fabricated by by magnetron sputtering in a Leybold Optics A550V7 sputter unit. Second, postdeposition oxidation in air transformed $\mathrm{YH}_{2}$ to $\mathrm{YHO}$. In order to achieve $\mathrm{YHO}$ upon air exposure, precursor $\mathrm{YH}_{2}$ films have to be deposited when the chamber pressure is above a certain critical value, which results in films with large structural disorder [21]. Further details on the synthesis process of photochromic YHO, both intrinsic and doped with $\mathrm{Zr}$, can be found elsewhere $[3,21,22]$. A cold white LED array from Thorlabs (color temperature 4600-9000 K) was used as an illumination source for the photodarkening experiments. The crystallographic structure of the obtained films was characterized by using $\mathrm{x}$-ray diffraction (XRD) in a Bruker Siemens D500 spectrophotometer $(\mathrm{Cu} K \alpha$ radiation, parallel beam geometry). The composition and surface oxidation states were studied by x-ray photoelectron spectroscopy (XPS) in an Ulvac PHI Quantera II instrument. Surface roughness characterizations were performed using atomic force microscopy with area of $5 \mu \mathrm{m}^{2}$ from a PhotonIc Technologies picostation. The optical transmittance $(T)$ of the YHO films in the clear and photodarkened state was measured using an Ocean Optics QE65000 spectrophotometer and a Perkin-Elmer Lambda 900 with an integrating sphere. Contact angle (CA) measurements were performed using a KSV Attension optical tensiometer under air. A 5- $\mu 1$ drop volume was used for each CA measurement, and three different sessile droplets were measured on several substrates for each value and averaged with a standard deviation of \pm 2 . CA values in the equilibrium $\left(\theta_{e}\right)$ for water, ethylene glycol (EG), and methylene iodide (MeI)—both EG and MeI from Sigma-Aldrich-were used to calculate surface free energies of yttrium oxyhydride films at clear and photodarkened state using the van Oss-Good-Chaudhury method [23,24].

The calculations were performed with the Vienna Ab Initio Simulation Package (VASP) code [25-27], based on density functional theory (DFT), using a plane-wave pseudopotential method together with the potential projector augmented wave (PAW) [28-30]. The generalized gradient approximation (GGA) in the scheme of Perdew-Burke-Ernzerhof (PBE) is used to describe the exchange-correlation functional [27]. To describe the electron-ion interaction standard PAW-PBE pseudopotentials [31] are used with $1 s^{1}$ for $\mathrm{H}, 2 s^{2} 2 p^{4}$ for $\mathrm{O}$, and $4 s^{2} 4 p^{6} 4 d^{1} 5 s^{2}$ for $\mathrm{Y}$ atoms as the valence-electron configuration. The plane-wave functions of valence electrons are expanded in a plane-wave basis set, and the use of PAW pseudopotentials allows a plane-wave energy cutoff $\left(E_{\text {cut }}\right)$. Only plane waves with kinetic energies smaller than $E_{\text {cut }}$ are used in the expansion. Reciprocal-space integration over the Brillouin zone is approximated through a careful sampling at finite number of $\boldsymbol{k}$ points using a MonkhorstPack mesh [30]. We choose the energy cutoff to be $700 \mathrm{eV}$, and the Brillouin-zone sampling mesh parameters for the $\boldsymbol{k}$-points set are $8 \times 8 \times 8$. In the optimization process the energy change is set to $1 \times 10^{-6} \mathrm{eV}$. The charge densities are converged to $1 \times 10^{-6} \mathrm{eV}$ in the self-consistent calculation. The range-separated hybrid Heyd-Scuseria-Ernzerhof (HSE06) functional is used for density-of-states calculations [32-34]. The hybrid functional requires a standard value of the (short-range) Hartree-Fock exchange (21\%) mixed with a portion of PBE exchange (79\%), also known as the HSE06 hybrid functional $[33,34]$. Selection of the parameter has been performed as an inverse value of infinity dielectric constant that is valid, if the energy band gap of these systems is larger than $3 \mathrm{eV}$.

\section{RESULTS AND DISCUSSION}

\section{A. Hydrophobicity control through light illumination}

YHO thin films exhibit photochromic properties, that is, YHO films undergo a reversible decrease of their optical transmittance when illuminated with light of adequate energy and intensity [22]. Figure 1(a) shows the transmittance in the clear and photodarkened state for a 1400-nm-thick YHO film. This film decreased its luminous transmittance $T_{\text {lum }}$ [35] from $78.5 \%$ to $26.7 \%$ after illumination. The luminous efficiency of the human eye (photopic vision) is presented in Fig. 1(a) for comparison [35]. How to obtain such optical contrast by illumination will be discussed in detail in the next section. Recent studies by Nafezarefi et al. [21] revealed that the bleaching dynamics and photochromic contrast in YHO is affected by $\mathrm{Zr}$ doping.

Nonilluminated (clear) YHO thin films show hydrophobicity with equilibrium contact angle $\theta_{e}$ values of $95^{\circ}$ for water (see Table I); however, $\theta_{e}$ values increased to $115^{\circ}$ after illumination (again in the case of water), see Table I and Fig. 1(b). Table I also shows $\theta_{e}$ for ethylene glycol EG and methylene iodine MeI for the clear and photodarkened states. In the case of MeI, $\theta_{e}$ also increases after illumination, from $43^{\circ}$ to $60^{\circ}$, yet remains constant for EG. Atomic force microscope AFM studies performed in such films revealed a relatively smooth surface with a rms value of surface roughness of around $8 \mathrm{~nm}$.

The observed initial hydrophobicity of the YHO films (clear state) can be explained by the electronic structure of rare-earth elements. According to a detailed experimental analysis of the entire rare-earth oxide series carried out by Azimi et al. [17], the unfilled $4 f$ orbitals shielded by a full octet 

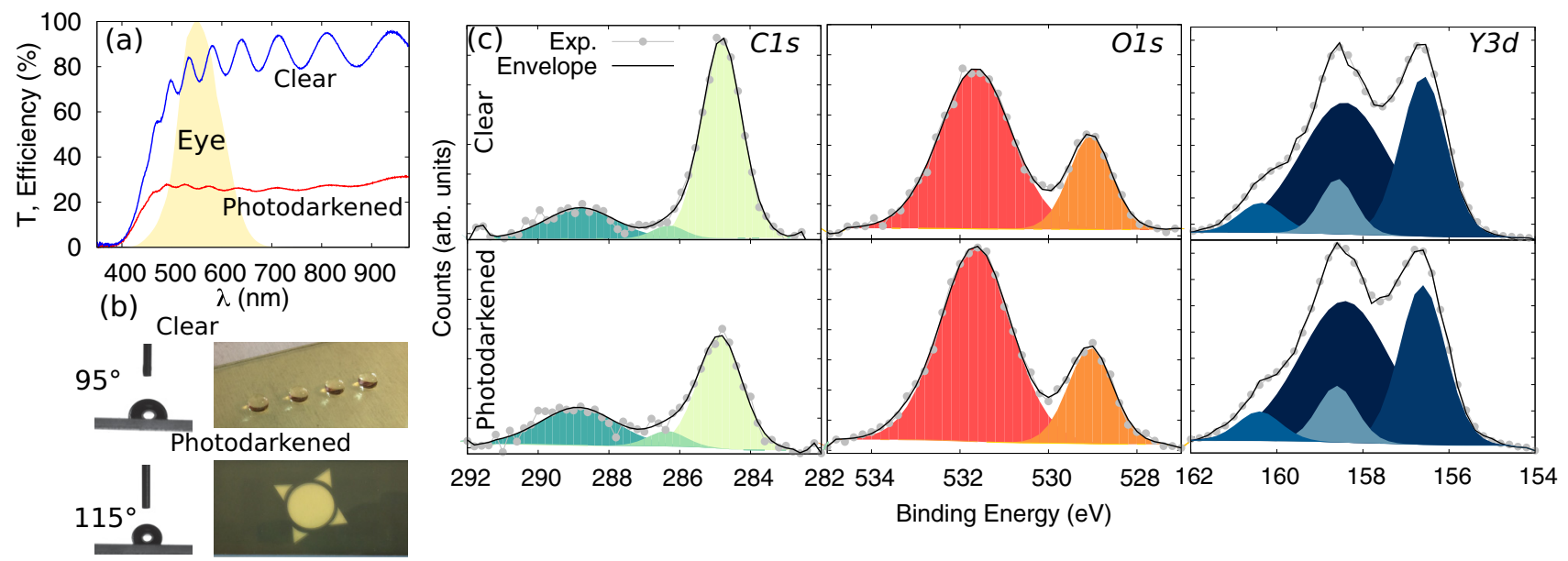

FIG. 1. Different properties measured in the clear state and photodarkened state (after illumination) for a 1400-nm-thick YHO film: transmittance compared to the luminous efficiency of the human eye in photopic vision [panel (a)], contact angle photographs for water, a photo of water droplets onto a clear film, as well as the same film after being illuminated under a sun-shaped mask during $1 \mathrm{~h}$ [panel (b)]. XPS spectra corresponding to $\mathrm{C} 1 s, \mathrm{O} 1 s$, and $\mathrm{Y} 3 d$ [panel (c)].

of electrons from the $5 s^{2} p^{6}$ shell result in a lower tendency of such compounds to form hydrogen bonds with the adjacent water molecules [17,36]. Hydrophobicity is not exclusive of the lanthanide $f$-shell group, but it can be achievable in any metal oxide provided that the electronegativity of such metal is low enough [16]. The low electronegativity of $\mathrm{Y}$ and the prevalence of yttrium oxide at the surface [37] explains the high $\theta_{e}$ shown in Table I.

One might expect decreased hydrophobicity under illumination caused by electron-hole pairs. Such behavior occurs in other metal oxides $[18,19,38-41]$. As we stated previously, however, hydrophobicity in YHO is enhanced under illumination. The light-induced decrease of wettability can be explained through changes in the oxygen-to-metal ratio at the surface. In metal oxides, coordinatively unsaturated oxygen atoms work as a Lewis base while the metal cations work as a Lewis acid. Combined Lewis acid and base orientation of the surface causes high affinity towards water molecules [42], therefore the oxygen-to-metal ratio in the surface is crucial for understanding the wettability properties [43].

In order to study the compositional changes of the YHO surface, XPS measurements were performed before and after illumination. The results are presented for $\mathrm{C} 1 s, \mathrm{O} 1 s$, and $\mathrm{Y} 3 d$ in Fig. 1(c). See Table II for the quantification of the different elements by XPS. The quantification has been done using survey spectra (not shown) considering $\mathrm{Y} 3 s, \mathrm{O} 1 s$, and $\mathrm{C} 1 s$ (column $\mathrm{A}$ ) or $\mathrm{Y} 3 p, \mathrm{O} 1 s$, and $\mathrm{C} 1 s$ (column $\mathrm{B}$ ) for both the clear and dark state.
The carbon (adventitious) $\mathrm{C} 1 s$ signal can be deconvoluted into three different contributions. The signal corresponding to $\mathrm{C}-\mathrm{C}$ has been established at $284.8 \mathrm{eV}$ as a charge correction reference. Other contributions are located at $286.3 \mathrm{eV}$ (attributed to $\mathrm{C}-\mathrm{O}-\mathrm{C}$ and/or $\mathrm{C}-\mathrm{OH}$, which are expected to present a $1.0-\mathrm{eV}$ difference in energy and hence are difficult to resolve), and $\mathrm{O}-\mathrm{C}=\mathrm{O}$ at $288.8 \mathrm{eV}$ [44].

After illumination, the carbon content on the surface decreases, see Table II. This decrease is more pronounced in the C-C contribution, Fig. 1(c). Since the content of C in the surface decreases, the increase of adsorbed hydrocarbons is ruled out as the possible cause for the light-induced enhancement of the hydrophobicity [45-47]. Nevertheless, a decrease in carbon content can result in an increase of the intensity of the XPS contributions located at higher energies [48]. In particular, the decrease of carbon may result in an increase of the O1s intensity when compared to the intensity of the $\mathrm{Y} 3 d$ signal. For this reason, $\mathrm{O} / \mathrm{Y}$ ratios in Table II have been calculated using $\mathrm{Y} 3 s$ and $\mathrm{Y} 3 p$ levels, which are closer in energy to $\mathrm{O} 1 s$ than $\mathrm{Y} 3 d$.

The O1s signal is composed of two contributions at 529.0 and $531.2 \mathrm{eV}$. The former can be attributed to $\mathrm{O}$ atoms bound to $\mathrm{Y}$ atoms, whereas the latter can be assigned to atomic oxygen [49]. After illumination, the oxygen-to-yttrium atomic ratio of the surface increases, see Table II. This increase is consistent when comparing the $\mathrm{O} 1 s$ level to $\mathrm{Y} 3 s$ and $\mathrm{Y} 3 p$ levels, and takes place both for $\mathrm{O}$ bound to $\mathrm{Y}$ as well as for atomic $\mathrm{O}$. The largest increase is observed in the latter,

TABLE I. Equilibrium contact angle values $\left(\theta_{e}\right)$ for water, ethylene glycol (EG), and methylene iodine (MeI), as well as the measured total surface energy $\left(\gamma_{\text {total }}\right)$ and its components: Lifshitz-van der Waals interactions term $\left(\gamma^{L W}\right)$ and acid-base interaction term $\left(\gamma^{A B}\right)-\operatorname{calculated}$ from the Lewis acid and base parameters $\left(\gamma^{+}\right.$and $\gamma^{-}$, respectively). All data given for the clear and photodarkened state.

\begin{tabular}{lccccccr}
\hline \hline & $\begin{array}{c}\theta_{e}(\text { Water }) \\
(\mathrm{deg})\end{array}$ & $\begin{array}{c}\theta_{e}(\mathrm{EG}) \\
(\mathrm{deg})\end{array}$ & $\begin{array}{c}\theta_{e}(\mathrm{MeI}) \\
(\mathrm{deg})\end{array}$ & $\begin{array}{c}\gamma_{\text {total }} \\
\left(\mathrm{mJ} / \mathrm{m}^{2}\right)\end{array}$ & $\begin{array}{c}\gamma^{L W} \\
\left(\mathrm{~mJ} / \mathrm{m}^{2}\right)\end{array}$ & $\begin{array}{c}\gamma^{A B} \\
\left(\mathrm{~mJ} / \mathrm{m}^{2}\right)\end{array}$ & $\begin{array}{c}\gamma^{+} \\
\left(\mathrm{mJ} / \mathrm{m}^{2}\right)\end{array}$ \\
\hline Clear state & 95 & 81 & 43 & 46.55 & 38.07 & 8.98 & 2.98 \\
Photodarkened state & 115 & 81 & 60 & 28.88 & 28.58 & 0.31 & 0.18 \\
\hline \hline
\end{tabular}


TABLE II. Quantification by XPS of the O, Y, and C content at the surface of a yttrium oxyhydride film in the clear and photodarkened state. Two sets of data are presented in each case. In case A the quantification has been done, taking into account levels $\mathrm{C} 1 s, \mathrm{O} 1 s$, and $\mathrm{Y} 3 s$, whereas in $\mathrm{B}, \mathrm{O} 1 s, \mathrm{C} 1 s$, and $\mathrm{Y} 3 p$ were considered instead.

\begin{tabular}{lcrrrr}
\hline \hline & \multicolumn{2}{c}{ Clear state } & & \multicolumn{2}{c}{ Dark state } \\
\cline { 2 - 3 } & $\mathrm{A}$ & $\mathrm{B}$ & & $\mathrm{A}$ & $\mathrm{B}$ \\
\hline $\mathrm{C}($ at \%) & 45.6 & 47.0 & 35.8 & 36.7 \\
$\mathrm{O}($ at \%) & 38.4 & 37.9 & & 48.1 & 46.9 \\
$\mathrm{Y}($ at \%) & 16.0 & 15.2 & & 16.2 & 16.4 \\
$\mathrm{O} / \mathrm{Y}$ & 2.4 & 2.5 & & 3.0 & 2.9 \\
$\mathrm{C} / \mathrm{Y}$ & 2.9 & 3.1 & & 2.2 & 2.2 \\
\hline \hline
\end{tabular}

Fig. 1(c). The contributions of the carbonates in the $\mathrm{O} 1 \mathrm{~s}$ region seem to be negligible.

The obtained results for $\mathrm{Y} 3 d$ correspond very well to the $\mathrm{Y}_{2} \mathrm{O}_{3}$ stoichiometry. At the surface the samples consist of $\mathrm{Y}_{2} \mathrm{O}_{3}$, which agrees with our previous work [37]. The $\mathrm{Y} 3 d$ has well-resolved spin-orbit components, namely, $\mathrm{Y} 3 d_{3 / 2}$ and $\mathrm{Y} 3 d_{5 / 2}$. These components can be deconvoluted into $\mathrm{Y}_{2} \mathrm{O}_{3}$, with contributions at 156.6 and $158.4 \mathrm{eV}$, Fig. 1(c) [49]. An extra doublet is needed for completing the fitting, with contributions at 158.6 and $160.3 \mathrm{eV}$. In this energy range, the possibilities are $\mathrm{Y}-\mathrm{OH}$ [50], yttrium carbonates [50], and $\mathrm{Y}-\mathrm{H}$ [51], the latter being the best candidate, since no evidence of carbonates and hydroxides is found in $\mathrm{C} 1 s$ or $\mathrm{O} 1 s$. There is not a remarkable change in $\mathrm{Y} 3 d$ before and after illumination.

When comparing the current XPS results with previous published data [52], it is evident that the films studied here, obtained by an optimized sputtering process [3], present higher homogeneity.

Surface-energy calculations, performed using the van OssChaudhury-Good method [23,24], confirm the lower wettability through reduction under illumination of the total surface energy $\gamma_{\text {total }}$, see Table I. The enrichment in oxygen of the surface, confirmed by XPS, reduces the Lewis sites as the surface approaches the $\mathrm{Y}_{2} \mathrm{O}_{3}$ stoichiometry. The nonpolar Lifshitz-van der Waals surface-energy component $\gamma^{L W}$ also decreases from 38.07 to $28.57 \mathrm{~mJ} / \mathrm{m}^{2}$, while the polar acidbase component $\gamma^{A B}$ decreases from 8.98 to $0.31 \mathrm{~mJ} / \mathrm{m}^{2}$ after illumination. Here $\gamma^{A B}=2\left(\gamma^{+} \gamma^{-}\right)^{1 / 2}$, where $\gamma^{+}$is the Lewis acid and $\gamma^{-}$the Lewis base parameters of surface tension.

Hydrophobic yttrium-based oxides have been reported in the past $[16,53]$. In those works, as $\mathrm{Y}_{2} \mathrm{O}_{3-x}$ coatings approached $\mathrm{Y}_{2} \mathrm{O}_{3}$ stoichiometries, contact angles increased [53]. This pattern is consistent with metal-to-oxygen ratios of surface studies [43]. Consequently, the enrichment in oxygen of the surface under illumination causes the light-induced hydrophobicity enhancement observed in YHO thin films. In the next section, the exchange of oxygen atoms between the film and the atmosphere, induced by illumination, is demonstrated.

\section{B. Light-induced breathing}

Photochromic yttrium oxyhydride has been obtained by the oxidation in air of reactively sputtered metallic $\mathrm{YH}_{2}$ thin films. The effect that the ambient humidity plays in this trans- formation is unclear. The incorporation of oxygen in the $\mathrm{YH}_{2}$ lattice causes the increase of the lattice constant $a$ from 5.20 to $5.34 \AA[2,3,54]$ and hence the displacement of the diffraction peaks towards lower angles. Under illumination, the lattice of the YHO films contracts back, but without reaching the original oxygen-free $\mathrm{YH}_{2}$ lattice constant [55].

After the incorporation of oxygen, NMR studies revealed that most of the hydrogen atoms in YHO remained in a local environment very similar to tetrahedral positions in $\mathrm{YH}_{2}$ [14]. Small signals, which can be attributed to mobile protons and to oxygen coordination, arise as well after air exposure.

Figure 2(a) shows a grazing incidence XRD pattern corresponding to a yttrium oxyhydride sample in its initial (clear), illuminated (photodarkened), and recovered (bleached) state. The standard diffraction peaks for $\mathrm{YH}_{2}$ and $\mathrm{Y}_{2} \mathrm{O}_{3}$ are also shown for comparison [Joint Committee of Powder Diffraction Standards (JCPDS) Cards No. 04-002-6938 and No. 04002-7545].

The analysis of the XRD patterns revealed how the films undergo an accordionlike transformation: the YHO lattice contracts and expands when subjected to illumination/darkness cycles.

Our previous optical studies pointed to the reversible formation of oxygen-deficient $\mathrm{YHO}_{1-x}$ metallic domains [13] within the dielectric YHO lattice as the cause of the photochromic behavior and lattice expansion/contraction:

$$
\mathrm{YHO} \underset{\text { darkness }}{\stackrel{\text { illumination }}{\rightleftharpoons}}(1-\eta) \mathrm{YHO}+\eta \mathrm{YHO}_{1-x}+\eta x \mathrm{O} .
$$

Since the filling factor $f f$ of the $\mathrm{YHO}_{1-x}$ domains is predicted to be very small [13], the factor $\eta$ must be $\eta \ll 1$. Dilution of $\mathrm{YHO}_{1-x}$ domains in the dielectric $\mathrm{YHO}$ structure is necessary to achieve higher optical absorption rather than higher optical reflectance, which is consistent with experimental observations [10,22].

Very few oxygen atoms need to be released under illumination to produce a large optical contrast. In fact, $f f=$ 0.02 causes a drop of the visible transmittance larger than $30 \%$ [22]. In addition, not all the released oxygen atoms necessarily need to leave the sample, and the material is able to host the outdiffused $\mathrm{O}$ atoms [56]. This was confirmed also by XPS in Fig. 1(c). Therefore, although the effective medium approximation works very well for modeling the optical properties [22], it is very difficult to confirm the release of oxygen in the experiment.

However, we postulate that there must be some exchange of oxygen atoms under illumination/darkness between the sample and its surroundings. This exchange, as discussed before, is probably below the detection limit of most conventional techniques. To prove our hypothesis, YHO thin films were subjected to 2-h-period cycles $(0.5 \mathrm{~h}$ illumination followed by $1.5 \mathrm{~h}$ darkness) inside a glovebox filled with $\mathrm{N}_{2}$. The $\mathrm{O}_{2}$ and $\mathrm{H}_{2} \mathrm{O}$ content within the glovebox was below 0.1 and $1.4 \mathrm{ppm}$, respectively. The average transmittance of the film was measured between 600 and $800 \mathrm{~nm}$ during cycling and plotted in Fig. 2(b). In the absence of air, the films lost part of their initial transparency in each cycle, not being able to recover fully. After 4 weeks of continuous cycling within the glovebox, the luminous transmittance $T_{\text {lum }}$ of the samples decreased from $78.5 \%$ in the nonilluminated state to $26.7 \%$. 

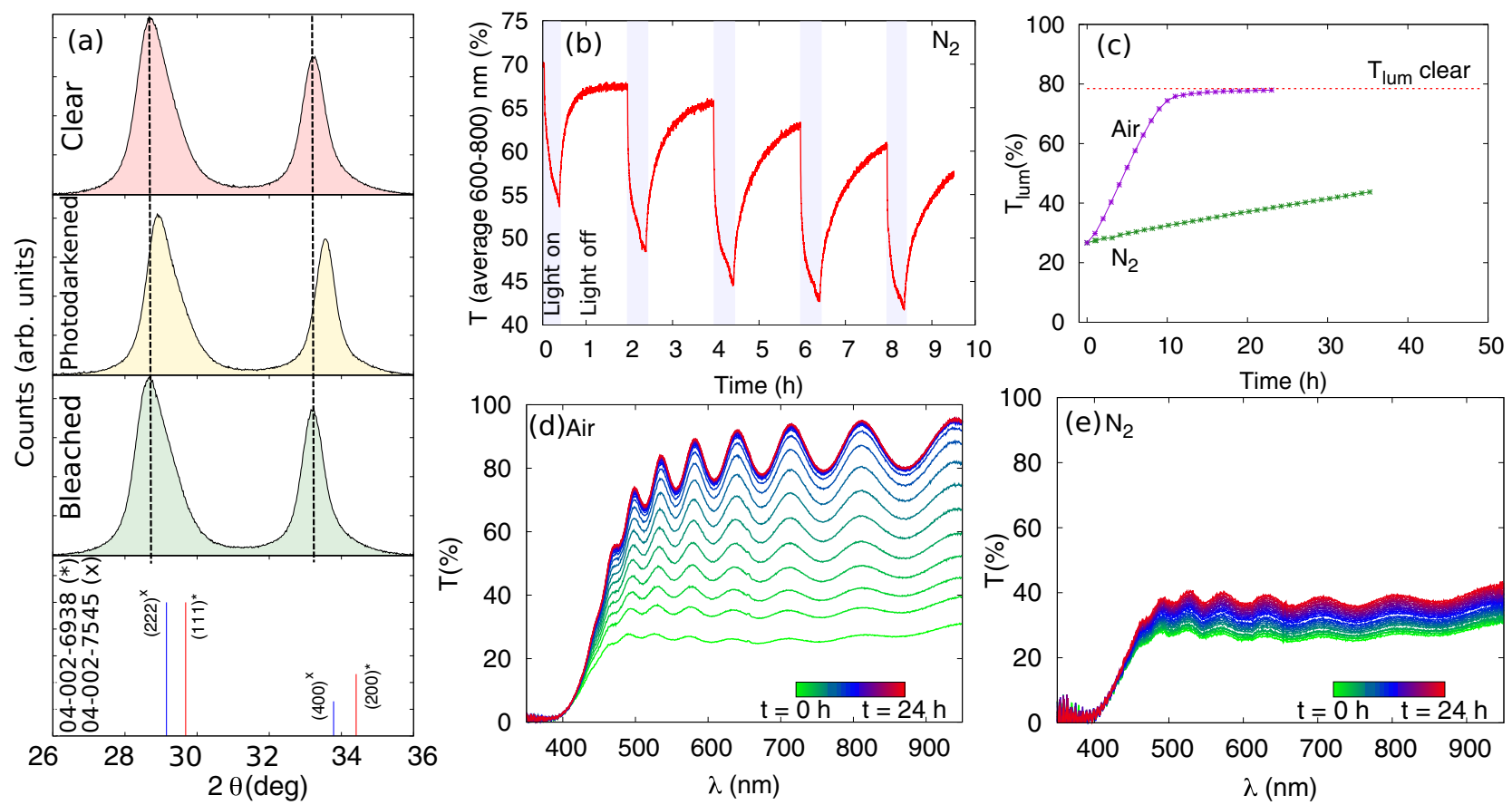

FIG. 2. Structural and optical changes under light illumination and the dependence of the photochromic dynamics on the atmosphere: x-ray diffraction patterns for a 1400-nm-thick YHO sample at the initial, dark, and bleached state [panel (a)]. Average transmittance measured between 600 and $800 \mathrm{~nm}$ during 0.5-h illumination followed by 1-h darkness in a sample kept in $\mathrm{N}_{2}$ atmosphere [panel (b)]. Differences in the recovery dynamics for the sample kept in air vs the sample kept in $\mathrm{N}_{2}$ atmosphere, as illustrated by the measurement of the luminous transmittance $T_{\text {lum }}$ vs time [panel (c)] and by a series of transmittance measurements vs time [panels (d) and (e)].

This heavily photodarkened films were allowed to bleach in total darkness, both in air and in $\mathrm{N}_{2}$ atmosphere (glovebox). The evolution of $\mathrm{T}_{\text {lum }}$ is presented in Fig. 2(c). The bleaching speed of the photodarkened samples in darkness was much slower inside the glovebox than in air. In addition, during the recovery, a series of transmittance measurements were performed during a period of $24 \mathrm{~h}$, both in air [Fig. 2(d)] and in the glovebox [ $\mathrm{N}_{2}$ atmosphere, Fig. 2(d)].

The films kept in air recover their initial transparency after a few hours $\left[\mathrm{T}_{\text {lum }}\right.$ clear, presented in Fig. 2(c) as a horizontal dashed line], while the films in $\mathrm{N}_{2}$ recovered very little in the same period of time. Since there are no significant differences between the temperature inside and outside of the glovebox (both at $\sim 20{ }^{\circ} \mathrm{C}$ ), the data presented in Figs. 2(c)2(e) strongly indicate that a source oxygen from the ambient is crucial for adequate recovering of the photodarkened films. The need for ambient oxygen, and possibly water vapor, is consistent with the light-induced oxygen release hypothesis summarized in Eq. (1).

The dependence on the atmospheric composition rules out other possible explanations for the photochromic mechanism, including light-induced formation of defect pairs or lattice distortion [14]. The release of hydrogen [15] (instead of oxygen) is an alternative explanation that can also be ruled out but for a different reason. In this case, the reversibility of the process would require the rehydrogenation of the film, a process that cannot take place at ambient pressure [1]. Nevertheless, if hydrogen is released, the film could bleach by the incorporation of $\mathrm{O}$, eventually approaching the $\mathrm{Y}_{2} \mathrm{O}_{3}$ stoichiometry. However, this hypothesis is not supported by the X-ray diffractograms shown in Fig. 2(a) and contradicts the reversibility of the process. Besides, the very large band gap of $\mathrm{Y}_{2} \mathrm{O}_{3}$ would lead to an increase of $T_{\text {lum }}$. Such increments are not observed, Figs. 2(c) and 2(d).

Considering the low electronegativity of $\mathrm{Y}$, the idea of oxygen being pushed out of the YHO lattice by illumination may seem counterintuitive at first. The thermodynamics and kinetics of Eq. (1) need further studies for clarification. In the next section, a preliminary theoretical model for understanding the light-induced oxygen release in YHO films is presented.

\section{Theoretical considerations}

The experimental evidence presented above points to lightinduced oxygen exchange between the film and the atmosphere. In the present section, this question is addressed by DFT modeling ( $a b$ initio calculations using VASP 5.3.5). It is known that photochromic YHO coatings are obtained experimentally by the partial oxidation of $\mathrm{YH}_{2}$ films in air. As discussed before, the incorporation of oxygen into $\mathrm{YH}_{2}$ results in the expansion of the $\mathrm{YH}_{2}$ lattice. The lattice parameter $a$ increases, and hence the XRD peaks corresponding to YHO appear displaced towards lower angles when compared to oxygen-free $\mathrm{YH}_{2}$, Fig. 3(a). The data presented in Fig. 3(a) corresponds to two different samples. The evolution of the XRD pattern for the same sample before, during, and after the $\mathrm{YH}_{2}$-to-YHO transformation can be found elsewhere [3].

The oxygen intake also causes the band-gap opening. Figure 3(b) shows the experimental transmittance $(T)$, reflectance $(R)$, and absorbance $(A)$ corresponding to $\mathrm{YH}_{2}$ and compared 

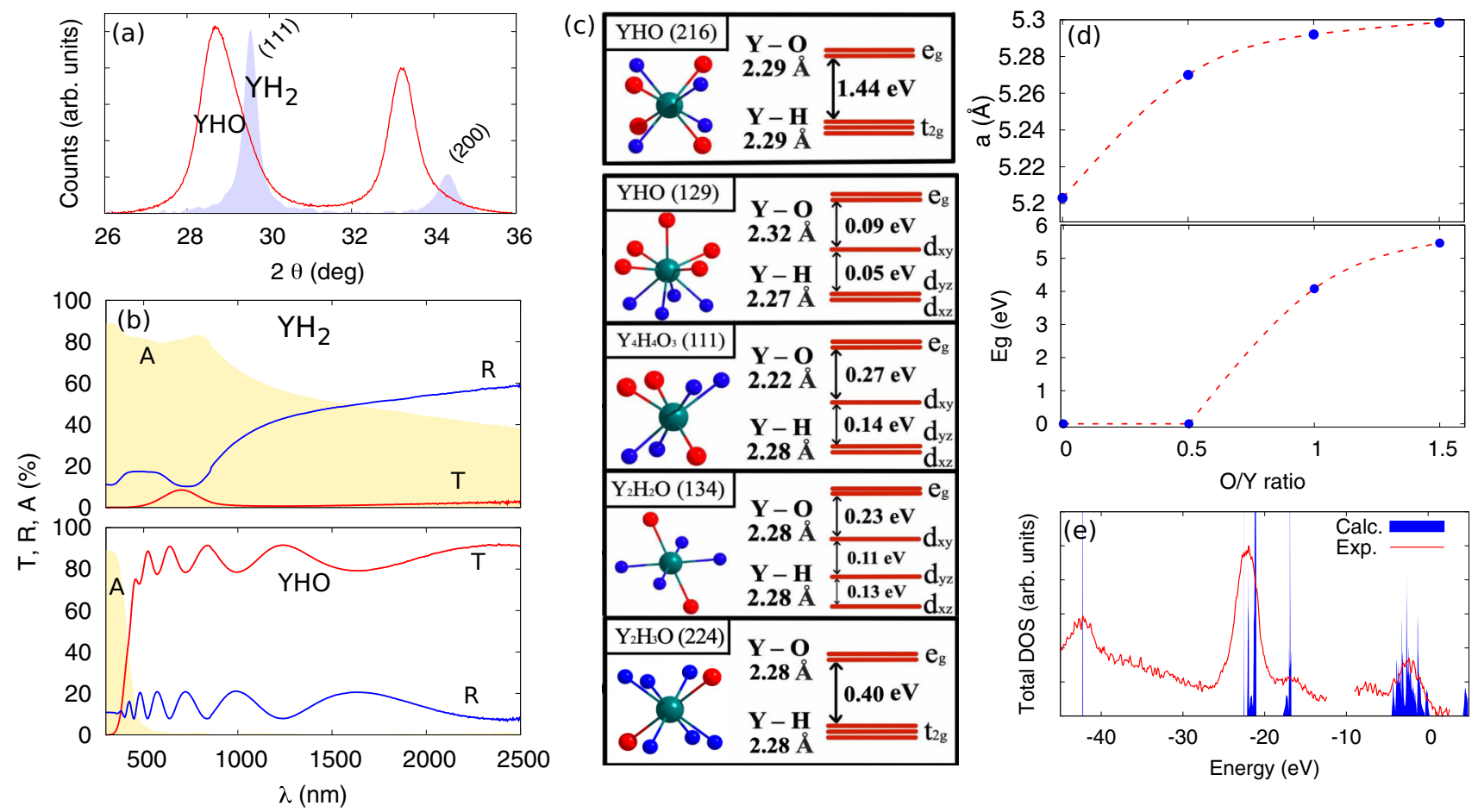

FIG. 3. XRD patters [panel (a)] and optical properties of transmittance $T$, reflectance $R$, and absorbance $A$ [panel (b)] corresponding to $\mathrm{YH}_{2}$ compared to photochromic yttrium oxyhydride (YHO). Schematic presentation of the bond coordination around $\mathrm{Y}$, Y-O, and Y-H bond lengths, and splitting of the $\mathrm{Y} 3 d$ states at the conduction-band minimum for $\mathrm{YHO}$ of different stoichiometries, $\mathrm{YH}_{x} \mathrm{O}_{y}$, where yttrium, hydrogen, and oxygen atoms are presented as green, blue, and red spheres, respectively [panel (c)]. Calculated lattice constant and band gap [panel (d)] as a function of $\mathrm{O} / \mathrm{Y}$ ratio in the $\mathrm{YH}_{x} \mathrm{O}_{y}$ system. Total density of states from $a b$ initio calculations as compared to experimental results from XPS measurements [panel (e)].

to photochromic $\mathrm{YHO}$. $\mathrm{YH}_{2}$ presents the optical behavior of a metal, but after the incorporation of oxygen it turns into YHO, a wide-band-gap semiconductor. The role played by the ambient humidity has not been studied yet.

Taking the crystalline structure of $\mathrm{YH}_{2}$ as the starting point $[f m-3 m$ and space group symmetry number (SPGN) 225], we build diverse $\mathrm{YHO}$ lattices of stoichiometry $\mathrm{YH}_{x} \mathrm{O}_{y}$, Fig. 3(c). In particular, multiscale modeling [57] predicted the possibility of lattices of stoichiometry (i) $\mathrm{Y}_{4} \mathrm{H}_{4} \mathrm{O}_{3} 111 P \overline{4} 2 m$ and (ii) $\mathrm{Y}_{2} \mathrm{H}_{2} \mathrm{O} 134 \mathrm{P} 42 / \mathrm{nmm}$ [57], as well as (iii) $\mathrm{YHO}$ and SPGN $129 P 4 / n m m, 215 P \overline{4} 3 m, 224 P n \overline{3} m$, and $216 P \overline{4} 3 m$.

Systematic theoretical and experimental studies [3] pointed to $P \overline{4} 3 m$ with SPGN 216 as the most energetically favorable yttrium oxyhydride lattice, i.e., stoichiometry $x=1$ and $y=1$ in $\mathrm{YH}_{x} \mathrm{O}_{y}$. In this structure, oxygen, as well as hydrogen atoms, are located in tetrahedral sites. The $x=1$ and $y=1$ stoichiometry is consistent with the recent exhaustive compositional experiments [37,58].

$\mathrm{YH}_{2}$ (225), $\mathrm{Y}_{4} \mathrm{H}_{6} \mathrm{O}_{2}$ (224), $\mathrm{Y}_{2} \mathrm{H}_{2} \mathrm{O}$ (134), as well as $\mathrm{Y}_{4} \mathrm{H}_{4} \mathrm{O}_{3}$ (111), present a metallic character, whereas $\mathrm{YHO}$ (216) is predicted to be a wide-band-gap semiconductor, as expected experimentally. According to these results, YHO crystallizes into a cubic structure with a lattice constant $a=$ $5.29 \AA$, which corroborates the lattice expansion that takes place in $\mathrm{YH}_{2}(a=5.20 \AA)$ when exposed to air. In particular, the expansion of $a$ and the opening of the band gap after air exposure is predicted by DFT, see Fig. 3(d). The calculated lattice constant and band gap are plotted as a function of the $\mathrm{Y} / \mathrm{O}$ ratio. The predicted value of $a$ for $\mathrm{YHO}$ is, however, slightly smaller than the experimental value observed ( $a=$ $5.34 \AA$ ) $[3,54]$. Discrepancies may arise from the difficulty in measuring $a$ due to the lattice strains, defects, or other deviations from ideality in these thin films.

The $\mathrm{Y}, \mathrm{O}$, and $\mathrm{H}$ atoms occupy the Wyckoff positions $4 c(1 / 4 ; 1 / 4 ; 1 / 4), 4 a(0,0,0)$, and $4 b(1 / 2,1 / 2,1 / 2)$, respectively, in this energetically favorable YHO (216) lattice. YHO belongs to the emerging family of materials called oxyhydrides [3]. The partial oxidation of $\mathrm{YH}_{2}$, and hence the formation of $\mathrm{YHO}$, triggers the expansion of the unit-cell volume [57]. As a consequence of the lattice expansion, the bond distances in YHO will be subjected to oxygen-induced elongation, Fig. 3(c). We show there the Y-O and Y-H bond lengths and the splitting of the $\mathrm{Y} 3 d$ states at the conductionband minimum for different $\mathrm{YH}_{x} \mathrm{O}_{y}$ stoichiometries.

The experimental XPS data and the calculated total density of states of YHO (216) are in good agreement, as shown in Fig. 3(c). The opening of a wide band gap as the oxygen atoms are incorporated in the $\mathrm{YH}_{2}$ structure is also predicted by the $a b$ initio calculations, Fig. 3(d). However, the model overestimates the band gap. The calculated value of $4.9 \mathrm{eV}$ for YHO (216) is about $1 \mathrm{eV}$ larger than the experimental band gap determined in the photochromic films by optical methods $[3,22]$.

It should be noted that the YHO films, obtained by the oxidation of $\mathrm{YH}_{2}$ previously prepared by reactive sputtering, are polycrystalline and multiphase in nature-note the widening of the XRD peaks of YHO when compared to $\mathrm{YH}_{2}$ in Fig. 3(a). Therefore, the energy-band diagram of the 
material most likely corresponds to a heterostructure of type II with a staggered band gap.

Assuming YHO (216) as the possible structure of photochromic yttrium oxyhydride, we can now explain the photochromic effect. The projected density of states (DOS) for YHO (216) revealed that both $\mathrm{O}$ and $\mathrm{H}$ atoms strongly contribute to the topmost valence band states. However, they are not hybridized because both $\mathrm{H}$ and $\mathrm{O}$ atoms are connected to the $\mathrm{Y}$ atoms independently from each other. On the other hand, the lowest conduction band is triply degenerate and formed mostly by Y $d$ states, in particular, $t_{2 g}$ states, Fig. 3(c). This result suggests that the light-induced $\mathrm{O}$ released from the film can be caused by the pseudo Jahn-Teller distortion effect: $\mathrm{Y}$ atoms are located at the center of the tetrahedral $\mathrm{H}$ and $\mathrm{O}$ sublattices. Under illumination, the transfer of electrons from the valence band to the $t_{2 g}$ bands will turn the YHO (216) lattice unstable [59].

As the $p$ orbitals of $\mathrm{O}$ atoms are hybridized with the $\mathrm{Y} d$ orbitals, the degeneracy of the $t_{2 g}$ states can be avoided by the removal of oxygen atoms. As a result, an O-deficient unit cell with smaller lattice constant will be created. As reported by Pishtshev et al. [57], there are many O-deficient structural arrangements that can be obtained from YHO, Fig. 3.

Before illumination, yttrium cations are in the oxidation state $3+$, which is the very stable state. After illumination, some (very few [13]) of the $\mathrm{O}$ atoms will be detached from the $\mathrm{Y}^{3+}$ cations. Those $\mathrm{Y}$ atoms evolve from a $3+$ to $2+$ oxidation state, which is less stable. In darkness, the $\mathrm{Y}^{2+}$ atoms oxidize back to $\mathrm{Y}^{3+}$ by the incorporation of oxygen atoms that remained within the lattice [56], Fig. 1(c), or newly incorporated from air.

As a result of illumination, metallic domains of smaller lattice constant will be created in the YHO (216) lattice, which results in the photochromic effect and the lattice contraction observed experimentally. The material seems to be able to host the out-diffused $\mathrm{O}$ atoms, which in some cases can reach the surface or even leave the film as demonstrated before. After stopping the illumination, the released $\mathrm{O}$ atoms can return to their former positions and the initial optical transparency will be restored.

\section{CONCLUSIONS}

When exposed to air the $\mathrm{YH}_{2}$ lattice expands from 5.20 to $5.34 \AA$ due to the incorporation of oxygen. In addition to the lattice expansion, $\mathrm{YH}_{2}$ turns into $\mathrm{YHO}$. YHO is transparent and photochromic. The reversibility of the photochromic effect depends on the surroundings of the films, being a source of oxygen necessary for the adequate bleaching of the samples. Therefore, the photochromic mechanism must involve oxygen diffusion and oxygen exchange between the sample and its surroundings. A consequence of the oxygen diffusion is the unusual enhancement of the hydrophobicity and the reversible lattice contraction of the YHO films under illumination. Although further studies are needed, a preliminary theoretical study points to the pseudo Jahn-Teller effect as the possible cause of this light-induced oxygen diffusion observed YHO films.

\section{ACKNOWLEDGMENTS}

This work has been supported by the Norwegian Research Council through the FRINATEK Project No. 287545, an internal project of the Institute for Energy Technology, and a Turkish Council of Higher Education Board 100/2000 Ph.D. scholarship. The computations were performed using the Norwegian Notur supercomputing facilities through Project No. nn4608k.

The authors contributed in the following ways-E.M.B: deposition of the YHO films, study of wettability, structural and optical properties of the YHO films; J.M.: deposition of the YHO films, study of structural and optical properties of the YHO films, cyclic optical measurements, XPS measurements, photochromic mechanism explanation, breathing concept, planning and formulation of conclusions; E.S.: DFT modeling of YHO. E.O.Z.: supervision on wettability measurements. S.K.: scientific supervision and support of the work, planning of experiments, mechanism of the effect, and formulation of conclusions; participation in DFT modeling. The paper was cowritten by J.M., E.M.B., and S.K. with the input of the rest of the authors
[1] J. N. Huiberts, R. Griessen, J. H. Rector, R. J. Wijngaarden, J. P. Dekker, D. G. D. Groot, and N. J. Koeman, Nature (London) 380, 231 (1996).

[2] F. Nafezarefi, H. Schreuders, B. Dam, and S. Cornelius, Appl. Phys. Lett. 111, 103903 (2017).

[3] J. Montero, F. A. Martinsen, M. Lelis, S. Z. Karazhanov, B. C. Hauback, and E. S. Marstein, Sol. Energy Mater. Sol. Cells 177, 106 (2018).

[4] M. La, N. Li, R. Sha, S. Bao, and P. Jin, Scr. Mater. 142, 36 (2018).

[5] A. Miniotas, B. Hjörvarsson, L. Douysset, and P. Nostell, Appl. Phys. Lett. 76, 2056 (2000).

[6] H. Kageyama, K. Hayashi, K. Maeda, J. P. Attfield, Z. Hiroi, J. M. Rondinelli, and K. R. Poeppelmeier, Nat. Commun. 9, 772 (2018).
[7] S. Cornelius, G. Colombi, F. Nafezarefi, H. Schreuders, R. Heller, F. Munnik, and B. Dam, J. Phys. Chem. Lett. 10, 1342 (2018).

[8] Y. Kobayashi, Y. Tsujimoto, and H. Kageyama, Annu. Rev. Mater. Res. 48, 303 (2018).

[9] Y. Kobayashi, Y. Tsujimoto, and H. Kageyama, Sci. Technol. Adv. Mater. 18, 905 (2017).

[10] T. Mongstad, C. Platzer-Björkman, J. P. Maehlen, L. P. A. Mooij, Y. Pivak, B. Dam, E. S. Marstein, B. C. Hauback, and S. Z. Karazhanov, Sol. Energy Mater. Sol. Cells 95, 3596 (2011).

[11] A. Ohmura, A. Machida, T. Watanuki, K. Aoki, S. Nakano, and K. Takemura, Appl. Phys. Lett. 91, 151904 (2007).

[12] A. Towns, Applied photochemistry, Applied Photochemistry: When Light Meets Molecules (Springer International Publishing, Cham, Switzerland, 2016), pp. 227-279. 
[13] J. Montero, F. A. Martinsen, M. García-Tecedor, S. Z. Karazhanov, D. Maestre, B. Hauback, and E. S. Marstein, Phys. Rev. B 95, 201301(R) (2017).

[14] C. V. Chandran, H. Schreuders, B. Dam, J. W. G. Janssen, J. Bart, A. P. M. Kentgens, and P. J. M. van Bentum, J. Phys. Chem. C 118, 22935 (2014).

[15] Q. Zhang, L. Xie, Y. Zhu, Y. Tao, R. Li, J. Xu, S. Bao, and P. Jin, Sol. Energy Mater. Sol. Cells 200, 109930 (2019).

[16] S. Zenkin, S. Kos, and J. Musil, J. Am. Ceram. Soc. 97, 2713 (2014).

[17] G. Azimi, R. Dhiman, H. M. Kwon, A. T. Paxson, and K. K. Varanasi, Nat. Mater. 12, 315 (2013).

[18] S. L. Ho, D. Kwak, Y. L. Dong, G. L. Seung, and K. Cho, J. Am. Chem. Soc. 129, 4128 (2007).

[19] X. Feng, L. Feng, M. Jin, J. Zhai, L. Jiang, and D. Zhu, J. Am. Chem. Soc. 126, 62 (2004).

[20] K. Yadav, B. R. Mehta, S. Bhattacharya, and J. P. Singh, Sci. Rep. 6, 35073 (2016).

[21] F. Nafezarefi, S. Cornelius, J. Nijskens, H. Schreuders, and B. Dam, Sol. Energy Mater. Sol. Cells 200, 109923 (2019).

[22] J. Montero and S. Z. Karazhanov, Phys. Status Solidi A 215, 1701039 (2018).

[23] C. J. van Oss, M. K. Chaudhury, and R. J. Good, Chem. Rev. 88, 927 (1988).

[24] C. J. van Oss, Colloids Surf., A 78, 1 (1993).

[25] G. Kresse and J. Furthmüller, Phys. Rev. B 54, 11169 (1996).

[26] G. Kresse and J. Furthmüller, Comput. Mater. Sci. 6, 15 (1996).

[27] G. Kresse and J. Hafner, Phys. Rev. B 49, 14251 (1994).

[28] P. E. Blöchl, Phys. Rev. B 50, 17953 (1994).

[29] G. Kresse and D. Joubert, Phys. Rev. B 59, 1758 (1999).

[30] G. Kresse and J. Hafner, Phys. Rev. B 47, 558 (1993)

[31] J. P. Perdew, K. Burke, and M. Ernzerhof, Phys. Rev. Lett. 77, 3865 (1996).

[32] J. Heyd, G. E. Scuseria, and M. Ernzerhof, J. Chem. Phys. 118, 8207 (2003).

[33] A. V. Krukau, O. A. Vydrov, A. F. Izmaylov, and G. E. Scuseria, J. Chem. Phys. 125, 224106 (2006).

[34] T. M. Henderson, J. Paier, and G. E. Scuseria, Phys. Status Solidi B 248, 767 (2011).

[35] S. Y. Li, G. A. Niklasson, and C. G. Granqvist, J. Appl. Phys. 108, 063525 (2010).

[36] N. Imanaka, in Binary Rare Earth Oxides, edited by G. Adachi, N. Imanaka, and Z.C. Kang (Springer, Dordrecht, 2004), p. 111.

[37] D. Moldarev, D. Primetzhofer, C. C. You, S. Z. Karazhanov, J. Montero, F. Martinsen, T. Mongstad, E. S. Marstein, and M. Wolff, Sol. Energy Mater. Sol. Cells 177, 66 (2018).
[38] X. Feng, J. Zhai, and L. Jiang, Angew. Chem. 44, 5115 (2005).

[39] S. Wang, X. Feng, J. Yao, and L. Jiang, Angew. Chem. 45, 1264 (2006).

[40] W. Zhu, X. Feng, L. Feng, and L. Jiang, Chem. Commun. 2753 (2006), doi: 10.1007/1-4020-2569-6.

[41] R.-D. Sun, A. Nakajima, A. Fujishima, T. Watanabe, and K. Hashimoto, J. Phys. Chem. B 105, 1984 (2001).

[42] H. H. Kung, Transition Metal Oxides, Studies in Surface Science and Catalysis Vol. 45 (Elsevier, The Netherlands, 1989), pp. 53-71

[43] S. Khan, G. Azimi, B. Yildiz, and K. K. Varanasi, Appl. Phys. Lett. 106, 061601 (2015).

[44] S. Yumitori, J. Mater. Sci. 35, 139 (2000).

[45] D. J. Preston, N. Miljkovic, J. Sack, R. Enright, J. Queeney, and E. N. Wang, Appl. Phys. Lett. 105, 011601 (2014).

[46] R. Lundy, C. Byrne, J. Bogan, K. Nolan, M. N. Collins, E. Dalton, and R. Enright, ACS Appl. Mater. Interfaces 9, 13751 (2017).

[47] E. Külah, L. Marot, R. Steiner, A. Romanyuk, T. A. Jung, A. Wäckerlin, and E. Meyer, Sci. Rep. 7, 43369 (2017).

[48] S. Evans, Surf. Interface Anal. 25, 924 (1997).

[49] V. Craciun, J. Howard, E. Lambers, R. Singh, D. Craciun, and J. Perriere, Appl. Phys. A 69, S535 (1999).

[50] M. Tellez-Cruz, M. Padilla-Islas, J. Godínez-Salomón, L. Lartundo-Rojas, and O. Solorza-Feria, Int. J. Hydrogen Energy 41, 23318 (2016).

[51] A. Fujimori and L. Schlapbach, J. Phys. C: Solid State Phys. 17, 341 (1984).

[52] T. Mongstad, A. Thøgersen, A. Subrahmanyam, and S. Karazhanov, Sol. Energy Mater. Sol. Cells 128, 270 (2014).

[53] H. C. Barshilia, A. Chaudhary, P. Kumar, and N. T. Manikandanath, Nanomaterials 2, 65 (2012).

[54] T. Mongstad, C. Platzer-Björkman, S. Karazhanov, A. Holt, J. Maehlen, and B. Hauback, J. Alloys Compd. 509, S812 (2011).

[55] J. P. Maehlen, T. T. Mongstad, C. C. You, and S. Karazhanov, J. Alloys Compd. 580, S119 (2013).

[56] H. W. Anes, Characterisation of YOH by TEM, Master's thesis, NTNU, Norwegian University of Science and Technology, 2017.

[57] A. Pishtshev, E. Strugovshchikov, and S. Karazhanov, Chemrxiv: 10.26434/chemrxiv.6950138.v1 (2018).

[58] D. Moldarev, M. V. Moro, C. C. You, E. M. Baba, S. Z. Karazhanov, M. Wolff, and D. Primetzhofer, Phys. Rev. Mater. 2, 115203 (2018).

[59] I. B. Bersuker, Chem. Rev. 113, 1351 (2013). 